\title{
Avaliação das alterações do sono em crianças com síndrome do respirador oral
}

\author{
Sleep changes evaluation in children with mouth breathing syndrome
}

Evaluación de las alteraciones del sueño en niños con síndrome del respirador oral

Caroline Feliz Fonseca Sepeda da Silva ${ }^{1 *}$, Vanessa Coutinho Aguiar Gomes ${ }^{1}$, Louise Silva e Silva Vilas Boas ${ }^{1}$, Angélica Cristina Pezzin¹.

\section{RESUMO}

Metodologia: trata-se de um estudo descritivo transversal e observacional, realizado no Hospital Universitário Bettina Ferro de Souza (HUBFS). Os dados foram colhidos através de um questionário. Resultados: Dentre os pacientes avaliados $57,9 \%$ eram do sexo masculino, na faixa etária de 5 a 10 anos $(57,4 \%)$, seguido de 3 a 4 anos $(21,5 \%)$. Em relação às manifestações verificadas, ronco $(84,6 \%)$, sono agitado $(75,4 \%)$, irritabilidade $(65,1 \%)$ e apneia $(60,5 \%)$ se mostraram os mais prevalentes, além da presença em praticamente metade dos pacientes $(46,7 \%)$ de comorbidades como rinite alérgica e asma. Conclusão: O sexo masculino e a faixa etária entre 5 a 10 anos são fatores importantes na suspeição de sintomas relacionados à SRO. $\mathrm{O}$ ronco como sintoma mais frequente durante o sono, demonstrando a ocorrência da respiração oral nestes pacientes, serve de alerta para os responsáveis e profissional de saúde na investigação e, consequente diagnóstico precoce da SRO.

Palavras-chave: Ronco, Sono, Criança, Otorrinolaringologia.

\begin{abstract}
Methods: This is a cross-sectional descriptive study, carried out at the Bettina Ferro de Souza Universitary Hospital. The collected data through a questionnaire. Results: Among the patients evaluated, $57.9 \%$ were male, in the age range of 5 to 10 years (57.4\%), followed by 3 to 4 years $(21.5 \%)$. In relation to the observed manifestations, snoring (84.6\%), restless sleep (75.4\%), irritability $(65.1 \%)$ and apnea $(60.5 \%)$ were the most prevalent, besides being present in almost half of patients (46.7\%) of comorbidities such as allergic rhinitis and asthma. Conclusion: The male gender and the age range between 5 and 10 years are important factors in the suspicion of ORS-related symptoms. The snoring as a more frequent symptom during sleep, demonstrating the occurrence of oral breathing in these patients, serves as an alert for those responsible and health professional in the investigation and consequent early diagnosis of ORS.
\end{abstract}

Keywords: Snoring, Sleep, Children, Otolaryngology.

\section{RESUMEN}

Metodología: se trata de un estudio descriptivo transversal y observacional, realizado en el Hospital Universitario Bettina Ferro de Souza (HUBFS). Los datos fueron recolectados a través de un cuestionario. Resultados: Entre los pacientes evaluados el 57,9\% eran del sexo masculino, en el grupo de edad de 5 a 10 años $(57,4 \%)$, seguido de 3 a 4 años $(21,5 \%)$. En cuanto a las manifestaciones verificadas, ronquido $(84,6 \%)$, sueño agitado $(75,4 \%)$, irritabilidad $(65,1 \%)$ y apnea $(60,5 \%)$ se mostraron los más prevalentes, además de la presencia en prácticamente la mitad de los pacientes $(46,7 \%)$ de comorbilidades como rinitis alérgica y asma. Conclusión: El sexo masculino y el grupo de edad entre 5 a 10 años son factores importantes en la sospectación de síntomas relacionados con la SRO. El ronquido como síntoma más frecuente durante el sueño, demostrando la ocurrencia de la respiración oral en estos pacientes, sirve de alerta para los responsables y profesional de salud en la investigación y, consecuente diagnóstico precoz de la SRO.

Palabras-clave: Ronquidos, Sueño, Niño, Otorrinolaringología.

${ }^{1}$ Universidade Federal do Pará. *E-mail: carol sepeda@hotmail.com

SUBMETIDO EM: $3 / 2019$

ACEITO EM: 4/2019

PUBLICADO EM: 7/2019

REAS/EJCH | Vol.Sup.24 | e637| DOI: https://doi.org/10.25248/reas.e637.2019 Página 1 de 7 


\section{INTRODUÇÃO}

A respiração oral é uma alteração funcional caracterizada pelo uso predominante da cavidade oral na respiração e o desuso da cavidade nasal podendo acometer indivíduos em qualquer faixa etária. Tal alteração, poderá ser consequência de um hábito ou obstrução nasal, ocasionada por congestão da mucosa nasal, e deformidades anatômicas das fossas nasais (RIBEIRO GCA, et al., 2016).

O respirador oral é um indivíduo que possui uma capacidade respiratória nasal restrita, que o leva a respirar pela boca na maior parte do tempo, tanto durante a vigília quanto no sono. Este quadro, quando perpetuado por um longo período, poderá promover diversas alterações anatômicas e funcionais, repercutindo em uma diversidade de sinais e sintomas, portanto, a clínica manifestada pelo paciente pode ser compreendida como uma síndrome, a Síndrome do Respirador Oral (SRO) (CAMPANHA SMA, et al., 2008).

No Brasil os dados epidemiológicos referentes à SRO são escassos. Entretanto, há algumas publicações evidenciando que 25 a $50 \%$ das crianças entre 8 e 10 anos apresentam respiração oral, e $26 \%$ entre 5 a 6 anos (PIGNATARI SSN e ANSELMO-LIMA LT, 2018).

Comumente, na SRO, pode-se observar alterações otorrinolaringológicas, craniofaciais e dentárias, nos órgãos fonoarticulatórios, corporais e nas funções orais. Dentre as alterações relacionadas ao crescimento facial, têm-se o aumento vertical do terço inferior da face, o estreitamento do arco maxilar, além do palato em ogiva, ângulo goníaco obtuso, má-oclusão - mordida aberta, dentes incisivos superiores em protrusão e mordida cruzada (CAMPANHA SMA, et al., 2008; MARSON A, et al., 2012; ALMEIDA FL, et al., 2009).

Nos lactentes e pré-escolares, as condições adquiridas, como hiperplasia adenoamigdaliana e os processos inflamatórios crônicos das vias aéreas superiores, são as causas obstrutivas mais frequentemente observadas, sendo que a hipertrofia irredutível adenoamigdaliana é considerada a forma primária das desordens respiratórias relacionadas ao sono, o que compromete o desenvolvimento físico e cognitivo infantil (POPOASKI C, et al., 2012; RIBEIRO ML e PINTO JA, 2006; EISER C e MORSE R, 2001; PIGNATARI SSN e ANSELMO-LIMA LT, 2018).

As consequências da respiração oral muito frequentemente são irreversíveis para o crescimento e desenvolvimento da criança causando: alterações posturais, craniofaciais, oclusais, auditivas, vocais, articulatórias; alterações nas funções estomatognáticas, nos órgãos fonoarticulatórios, na redução de apetite; dificuldades de atenção e concentração, agitação, ansiedade e dificuldade na aprendizagem, desempenho inferior de habilidades fonológicas e envelhecimento facial precoce em comparação aos respiradores nasais.

Destaca-se ainda a maior presença de olheiras, pregas palpebrais inferiores, aprofundamento do sulco mentolabial, face discretamente alongada na região das bochechas e maior desproporção facial (NISHIMURA CM e GIMENEZ SRML, 2010; FILUS JF, 2006; BRASILEIRO H, 2009).

Pacientes com SRO podem evidenciar dificuldades gerais de aprendizagem, ou seja, atraso em aritmética, leitura, escrita, transcrição e interpretação de texto, se comparados a alunos respiradores nasais. Nas atividades de interpretação de texto e de resolução de problemas aritméticos, o baixo desempenho dos respiradores orais pode ser decorrente da dificuldade de compreensão - $82 \%$ e $85 \%$ dos escolares não conseguiram interpretar de maneira correta, os enunciados dos problemas e da história lida, respectivamente (BRASILEIRO H, 2009).

Entretanto, é importante salientar que a compreensão dos determinantes da aprendizagem e de seu fracasso é multifatorial e complexa, e poucos são os instrumentos validados para investigação dos distúrbios de aprendizagem (RIBEIRO GCA, et al., 2016).

A conexão entre a SRO e o distúrbio respiratório do sono é clara, sendo este associado ao aumento de tonsilas faríngea e palatinas, com manifestação variando desde o ronco primário até a apneia obstrutiva do sono, com pico entre 2 e 6 anos de idade.

Há relatos de que no Brasil, as crianças em idade escolar apresentem uma prevalência de ronco habitual de $27,6 \%$, sonolência diurna excessiva de $7,8 \%$ e de apneia em $0,8 \%$, sendo estes os principais indicadores de distúrbios do sono (PIGNATARI SSN e ANSELMO-LIMA WT, 2018; GODOLFIM LF, 2010). 
A síndrome da apneia e hipopneia obstrutiva do sono (SAHOS) é caracterizada pela ocorrência repetitiva de obstrução total (apneia) ou parcial (hipopneia) da via aérea superior durante o sono. Tais obstruções levam à redução da oferta de oxigênio, e, consequentemente, a microalterações isquêmicas no tecido neural. Fisiologicamente, tais manifestações levam à ocorrência de despertares noturnos que objetivam a melhora da oxigenação a níveis aceitáveis, levando à fragmentação do sono (ZANCANELLA E, 2010).

A SAHOS pode ocorrer em crianças de todas as faixas etárias, com maior incidência entre 3 e 5 anos (ALENCAR HBB, et al., 2016). É considerado um distúrbio respiratório crônico, progressivo, incapacitante, de alta morbimortalidade, tendo como principais consequências os distúrbios comportamentais (hiperatividade, déficit de atenção, agressividade e comportamento opositor), déficit de aprendizado, hipertensão pulmonar e o prejuízo do crescimento somático (MARTINS AB, et al., 2007; BASS JL, 2004).

Apesar de bem documentados os efeitos negativos que as alterações relacionadas ao sono podem causar na saúde e no desenvolvimento da criança com respiração predominantemente oral, nota-se uma falta do devido rastreio destes indivíduos, levando ao subdiagnóstico clínico. Tal déficit torna importante o desenvolvimento deste estudo, com o objetivo da identificação dos aspectos clínicos relacionados aos principais sinais e sintomas sugestivos de alterações do sono em crianças em um serviço de referência. $O$ qual poderá servir de base para a orientação de profissionais de saúde e para o diagnóstico e tratamento precoces dos distúrbios do sono na criança e suas alterações.

\section{MÉTODO}

A pesquisa foi aprovada pelo Comitê de Ética em Pesquisa CAAE 43384915.9.0000.0018, parecer $n^{\circ}$ 1.097.646, em junho de 2016, sendo respeitadas as Normas de Pesquisa envolvendo seres humanos, conforme a Resolução no466 de 12/12/2012 do Conselho Nacional de Saúde. Os responsáveis pelas crianças preencheram o Termo de Consentimento Livre e Esclarecido (assinado pelos responsáveis das crianças entre cinco e 12 anos incompletos) e Termo de Assentimento Livre e Esclarecido (assinado pelos próprios pacientes, se tivessem 12 anos completos).

Trata-se de um estudo descritivo, prospectivo, transversal e observacional, no qual obteve-se uma amostra de 195 pacientes, com idade variando entre zero a 12 anos. A coleta dos dados foi realizada através do preenchimento de questionário apresentando perguntas direcionadas e constatou de dados como gênero, idade, informações sobre sinais e sintomas da SRO. A pesquisa foi realizada no setor de Otorrinolaringologia e Fonoaudiologia do HUBFS, localizado na cidade de Belém-PA, no período de março de 2016 a setembro de 2018.

As informações da caracterização amostral foram apuradas em banco de dados elaborado no software Microsoft ${ }^{\circledR}$ Office Excel ${ }^{\circledR}$ 2010. A estatística analítica foi utilizada para avaliar os resultados das variáveis categóricas da amostra através dos Testes $\mathrm{G}$ ao obter números menores que 5 e Qui-Quadrado de Aderência para tabelas univariadas. As estatísticas descritiva e analítica, foram realizadas no software BioEstat ${ }^{\circledR}$ 5.3. Para a tomada de decisão, adotou-se o nível de significância $p=0,05$ ou $5 \%$, sinalizando com asterisco $\left({ }^{*}\right)$ os valores significantes.

\section{RESULTADOS}

Em relação ao sexo dos pacientes, verificou-se que do total de $195,113(57,9 \%)$ eram do sexo masculino e $82(42,1 \%)$ eram do sexo feminino. Segundo o teste Qui-Quadrado Aderência, houve incidência significante de pacientes do sexo masculino (Tabela 1).

Tabela 1 - Frequência quanto ao sexo na avaliação das alterações do sono em crianças com síndrome do respirador oral.

\begin{tabular}{ccc}
\hline Sexo & Frequência & $\%$ \\
\hline Masculino* $^{*}$ & 113 & $57,9 \%$ \\
Feminino & 82 & $42,1 \%$ \\
\hline Total & $\mathbf{1 9 5}$ & $\mathbf{1 0 0 , 0 \%}$ \\
\hline
\end{tabular}

Fonte: Dados de Pesquisa, 2019.

${ }^{*} \mathrm{p}<0.0001$ Teste Qui Quadrado Aderência. 
Observou-se neste estudo uma frequência significante de pacientes na faixa etária de 5 a 10 anos $(57,4 \%)$. Nas demais faixas etárias verificou-se valores inferiores, a exemplo de 0 a 2 anos (6,7\%), 3 a 4 anos (21,5\%) e 11 a 12 anos (14,4\%) (Gráfico 1).

Gráfico 1 - Frequência da faixa etária na avaliação das alterações do sono em crianças com síndrome do respirador oral.

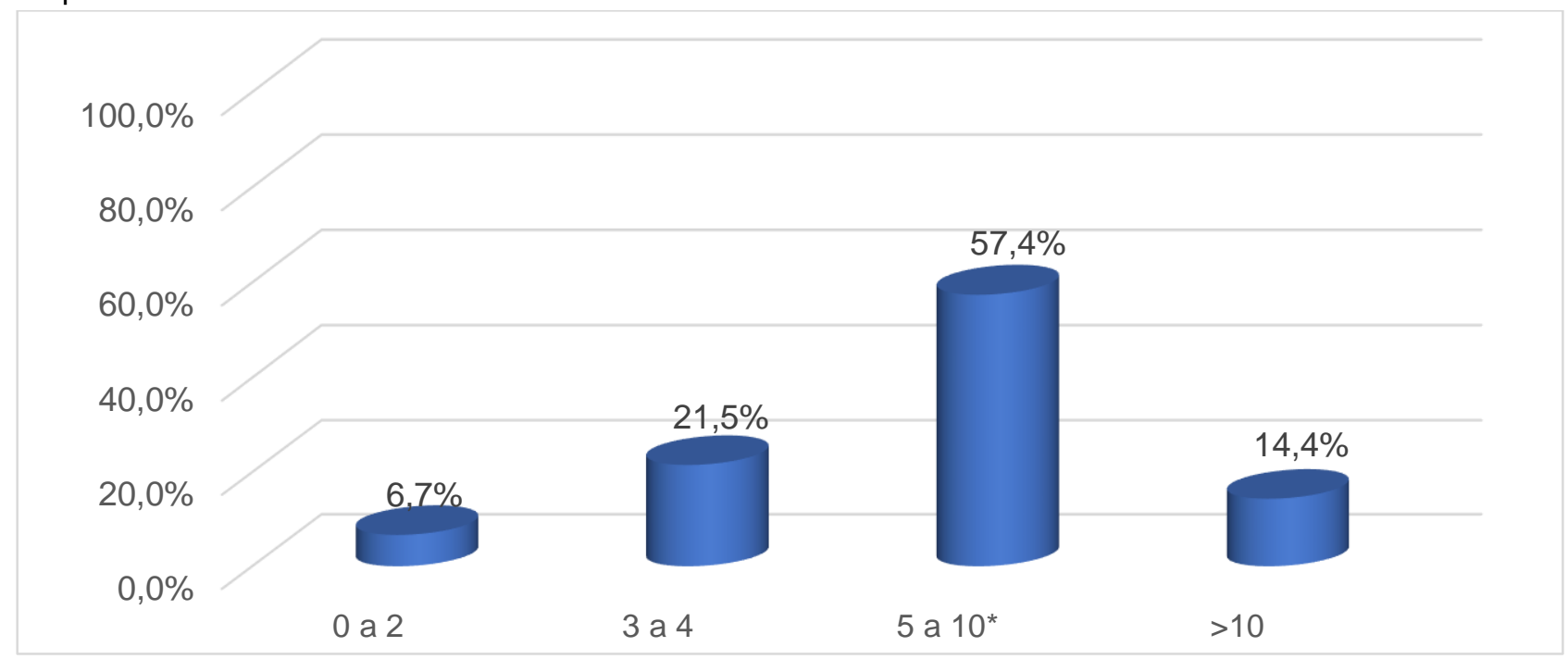

${ }^{*} p<0.0001$ Teste Qui-Quadrado Aderência

Fonte: Dados de Pesquisa, 2019

$\mathrm{Na}$ avaliação da anamnese dos pacientes entrevistados, quando questionados sobre a presença de sinais e sintomas durante o sono, ou em consequência dele, percebeu-se que os roncos (84,6\%), sono agitado (75,4\%), irritabilidade $(65,1 \%)$ e apneia $(60,5 \%)$ foram os mais prevalentes (Gráfico 2 ).

Gráfico 2 - Incidência dos sinais e sintomas apresentados pelos pacientes na avaliação das alterações do sono em crianças com síndrome do respirador oral.

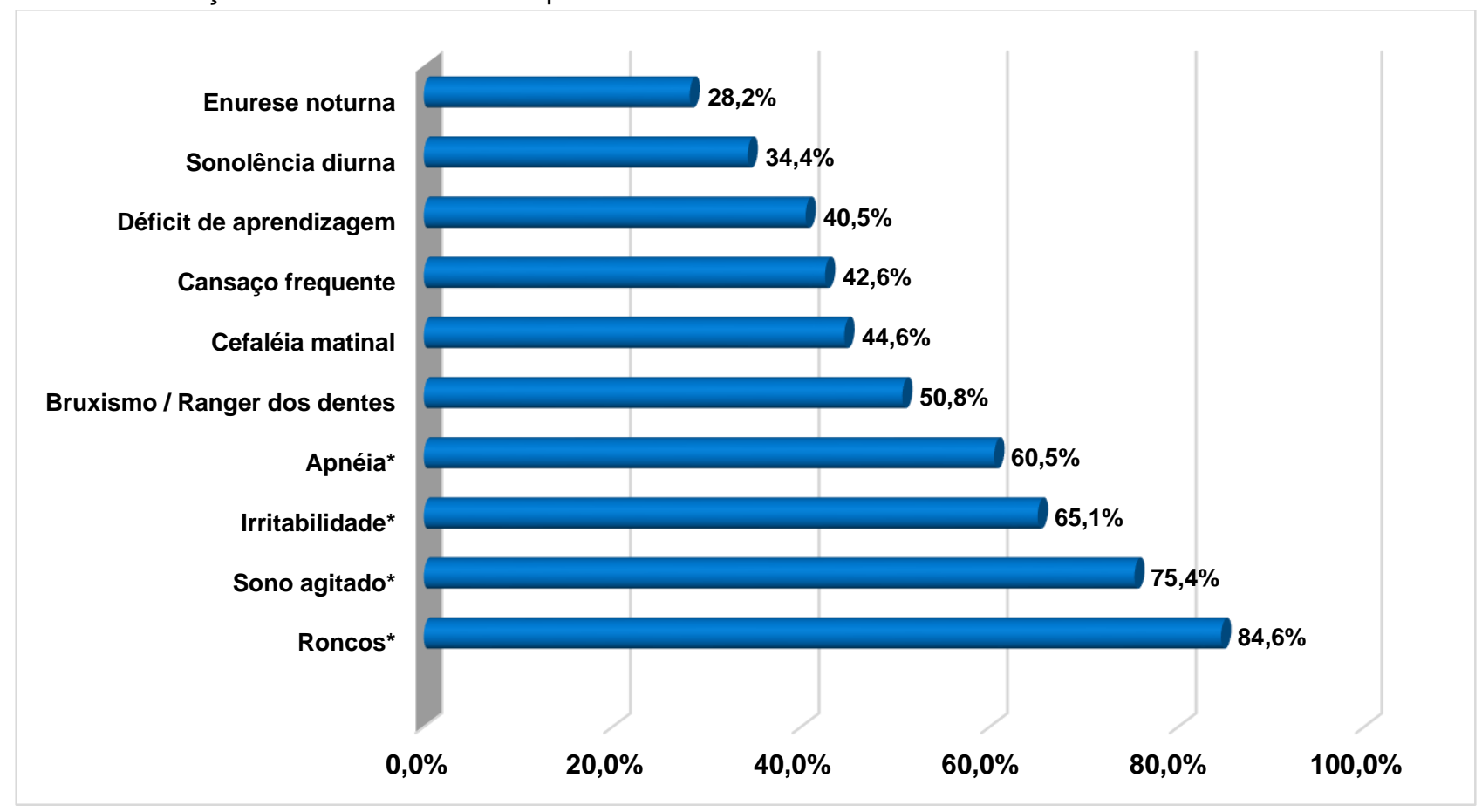

${ }^{*} \mathrm{p}<0.05$ Teste G Aderência

Fonte: Dados de Pesquisa, 2019. 
Constatou-se que, das 195 crianças, 91 (46,7\%) apresentavam alguma comorbidade, e que das referidas, observou-se a presença de rinite alérgica em 71 (36,4\%), e de asma em 20 (10,2\%) pacientes (Tabela 2).

Tabela 3 - Distribuição das comorbidades e seus principais tipos na avaliação das alterações do sono em crianças com síndrome do respirador oral.

\begin{tabular}{lcc}
\hline \multicolumn{1}{c}{ Comorbidades } & Frequência & \% (N = 195) \\
\hline Possui & & \\
Presença* $^{*}$ & 91 & $46,7 \%$ \\
Ausência & 71 & $36,4 \%$ \\
Desconhecido & 40 & $32,3 \%$ \\
Quais comorbidades & & $\mathbf{N}=91$ \\
Rinite alérgica* & 71 & $78 \%$ \\
Asma & 20 & $22 \%$ \\
\hline
\end{tabular}

${ }^{\star} p<0.0001$ Teste Qui-Quadrado Aderência

Fonte: Dados de Pesquisa, 2019.

\section{DISCUSSÃO}

As consequências da SRO muitas vezes são irreversíveis para o crescimento e desenvolvimento da criança (NISHIMURA CM e GIMENEZ SRML, 2010). Na literatura é possível observar e, de certa forma, prever os possíveis desfechos para os portadores e, nesse sentido, é imprescindível que os profissionais que lidam com crianças saibam identificar corretamente a presença de sinais e sintomas da síndrome, ainda que apenas por relatos dos pais e responsáveis. Neste estudo, foram avaliados diversos aspectos para compreender melhor a população de respiradores orais em nossa região.

Sabe-se que são diversas as alterações que podem ser percebidas em um indivíduo respirador oral. Alguns exemplos são as alterações posturais e de estruturas do sistema estomatognático, que acarretam distúrbios no crescimento dentofacial, sucção, mastigação, deglutição e fala, podendo inclusive evoluir para doença cardiorrespiratória e endocrinológica, distúrbios do sono, do humor e déficits do desempenho escolar (ABREU RR, et al., 2008). Portanto, no que tange tais alterações, evitar o seu desenvolvimento é crucial.

No presente estudo, pode-se observar o predomínio do sexo masculino entre os pacientes entrevistados, achado também verificado por Di Francesco RC, et al. (2004) e Menezes VA, et al. (2006). O sexo masculino é um fator de risco para diversas entidades nosológicas na pediatria e na otorrinolaringologia (PIGNATARI SSN e ANSELMO-LIMA WT, 2018), sendo uma característica fenotípica também importante de se considerar durante ananmnese. Apenas o estudo de Felcar JM, et al. (2010) destoou dos demais trabalhos presentes na literatura, apresentando um predomínio do sexo feminino.

Com relação a faixa etária das crianças avaliadas, o presente estudo apresentou uma frequência maior na idade de 5 a 10 anos, dado este que corrobora com os dados apresentados por Abreu RR et al, que encontrou uma média de idade de 5,9 anos $( \pm 1,9)$ (ABREU RR, et al., 2008) e também em demais literaturas (PIGNATARI SSN e ANSELMO-LIMA WT, 2018), justificada pela fisiologia do crescimento tecidual linfático na infância e no maior desenvolvimento do sistema imunológico com a substituição progressiva da imunidade passiva recebida na vida intrauterina pela imunidade ativa desenvolvida através da estimulação pelo contat com o ambiente. Vale ressaltar que as obstruções das vidas aéreas também têm reforço pelas alterações anatômicas e pelas próprias dimensões das paredes das vias aéreas, altamente influenciadas pela imunologia e pelo desenvolvimento tecidual. $\mathrm{Na}$ análise da escolaridade, este trabalho obteve como resultado uma alta frequência de crianças cursando o ensino fundamental nos anos iniciais. Dado este que reafirma aquele encontrado por Popoaski C, et al. (2012), sendo compatível também com relação à idade.

Sabe-se que crianças institucionalizadas e que frequentam escolas estão mais sujeitas a repetição de episódios de infecções de vias aéreas superiores, acarretando em proliferação dos tecidos linfoides e levando a obstruções nasais, roncos e respiração oral, perpetuando um ciclo fisiopatológico de novas infecções e 
piora das obstruções (PIGNATARI SSN e ANSELMO-LIMA WT, 2018). Na análise da anamnese (sinais e sintomas), as manifestações mais frequentes foram a presença de ronco, sono agitado, irritabilidade e apneia, dados estes que estão de acordo com aqueles encontrados nas principais literaturas (DI FRANCESCO RC, et al., 2004; MENEZES VA, et al., 2006; COSTA M, et al., 2015), estando os roncos e o sono agitado em posição de destaque.

Todos esses sinais e sintomas podem estar presentes nos portadores da SRO, devendo sempre serem questionados ativamente durante exame clínico. O protocolo deste estudo pode ser utilizado como instrumento de rastreio para profissionais atendentes do público pediátrico, ressaltando que os sinais e sintomas em conjunto aumentam a especificidade do quadro, pois é possível diagnóstico diferencial em pacientes com outras condições crônicas como a paralisia cerebral (VIANNA CIO e SUZUKI HS, 2011).

Ainda que sem relevância estatística na amostra deste estudo, sintomas como enurese noturna, cefaleia matinal, déficit de aprendizagem e cansaços frequentes diurnos são sintomas componentes das consequências da síndrome da apneia obstrutiva do sono e da hipóxia central, fazendo parte da sua complexa fisiopatologia, aumentando a possibilidade diagnóstica. Tais manifestações também devem ser investigados durante a anamnese, haja visto que como podem ser tidas como alterações comportamentais pelos responsáveis e se confundir com manifestações de outras condições, pois a respiração oral é complexa e traz à tona implicações que não envolvem apenas o estado de saúde do paciente (TERSE-RAMOS R, 2013).

No quesito comorbidades e seus tipos, o este estudo encontrou relação com quadros de rinite alérgica e asma, tal como Craig TJ, et al. (2005) que descreveram que a associação de asma e rinite alérgica no SRO são de tal importância que alguns preferem o termo "doença de vias aéreas unidas". Popoaski C, et al. (2012) descreveram em seu estudo que a chance de se encontrar um indivíduo asmático foi quase 8 vezes maior do que no grupo controle, e verifica-se que esta prevalência é altamente encontrada na literatura (RIBEIRO ML e PINTO JA, 2006; ABREU RR, et al., 2008). O presente estudo encontrou forte associação de comorbidades influenciando na qualidade do sono das crianças entrevistadas. Salienta-se que houve significância com a presença de rinite alérgica. A rinite alérgica, em sua fisiopatologia, também contribui para a obstrução das vias aéreas por edema da mucosa nasal, também devendo ser investigada e diagnosticada, haja visto de fala-se de uma doença crônica tratável clinicamente e que depende de manutenção para educação e controle ambiente em casa e na escola, além de ser uma das entidades mais comuns na clínica otorrinolaringológica (PIGNATARI SSN e ANSELMO-LIMA WT, 2018).

\section{CONCLUSÃO}

Demonstrou-se que o sexo masculino possui maior frequência entre os pacientes com a SRO, e que a faixa etária mais prevalente se apresenta entre 5 a 10 anos de idade. Além disso, foi possível verificar que, dentre as alterações mais observadas pelos pais, a mais prevalente durante o sono foi a respiração bucal. Tal fato corrobora para a necessidade de suspeição e investigação precoce da doença, de forma a evitar repercussões deletérias para a população pediátrica, perfil este em franco desenvolvimento físico e psíquico. $\mathrm{Em}$ associação, futuros estudos tornam-se necessários para que esta investigação possa se tornar um instrumento capaz de identificar e avaliar não somente as alterações físicas, mas também o impacto na qualidade de vida dos pacientes respiradores orais.

\section{REFERÊNCIAS}

1. ABREU RR, ROCHA RL, LAMOUNIER JA, et al. Prevalência de crianças respiradoras orais. Jornal de Pediatria, 2008; 84(5): 467-470.

2. ALENCAR HBB, OLIVEIRA MT, CARAM JM, et al. Tratamento da Síndrome da Apneia Obstrutiva do Sono com o Uso Supervisionado de Aparelhos Intraorais: Relato de Caso. Ensaios e Ciência: Ciências Biológicas, Agrárias e da Saúde, 2016, 20(2): 59-64.

3. ALMEIDA FL, SILVA AMT, SERPA EO. Relação entre má oclusão e hábitos em respiradores orais. Revista CEFAC, 2009; 11(1): 86-93.

4. BASS JL, CORWIN M, GOZAL D, et al. The effect of chronic or intermittent hypoxia on cognition in childhood: a review of the evidence. Pediatrics, 2004; 114(3): 805-816. 
5. BRASILEIRO H. Síndrome da apneia e hipopneia obstrutiva do sono - SAHOS. Revista da Faculdade de Ciências Médicas de Sorocaba, 2009; 11(1): 1-3.

6. CAMPANHA SMA, FREIRE LMS, FONTES MJF; O impacto da asma, da rinite alérgica e da respiração oral na qualidade de vida de crianças e adolescentes. Revista CEFAC, 2008; 10(4): 513-519.

7. CAMPOSTRINI DDA, PRADO LDF, PRADO GF. Síndrome da apneia obstrutiva do sono e doenças cardiovasculares. Revista Neurociências, 2014; 22(1): 102-112.

8. CHAVES JUNIOR CM, DAL-FABBRO C, BRUIN VMS, et al. Consenso brasileiro de ronco e apneia do sono aspectos de interesse aos ortodontistas. Dental Press Journal of Orthodontics, 2011; 16(1): 34.e1-e10.

9. COSTA M, VALENTIM AF, BECKER HMG, et al. Achados da avaliação multiprofissional de crianças respiradoras orais. Revista CEFAC, 2015; 17(3): 864-878.

10. CRAIG TJ, HANKS CD, FISHER LH. How do topical nasal corticosteroids improve sleep and daytime somnolence in allergic rhinitis? Journal of Allergy and Clinical Immunology, 2005; 116(6): 1264-1266.

11. DI FRANCESCO RC, PASSEROTTI G, PAULUCCI B, et al. Respiração oral na criança: repercussões diferentes de acordo com o diagnóstico. Revista Brasileira de Otorrinolaringologia, 2004; 70(5): 665-670.

12. EISER C, MORSE R. The measurement of quality of life in children: past and future perspectives. Journal of Developmental \& Behavioral Pediatrics, 2001; 22(4): 248-255.

13. EPSTEIN LJ, KRISTO D, STROLLO PJ, et al. Clinical guideline for the evaluation, management and long-term care of obstructive sleep apnea in adults. Journal of Clinical Sleep Medicine, 2009; 5(3): 263-276.

14. FELCAR JM, BUENO IR, MASSAN ACS, et al. Prevalência de respiradores bucais em crianças de idade escolar. Ciência \& Saúde Coletiva, 2010; 15(2): 437-444.

15. FILUS JF. Estudo de problemas posturais e de aprendizagem em alunos respiradores orais, PR. Dissertação (Mestrado em Educação - Universidade Estadual de Maringá, 2006; 97p.

16. GODOLFIM LR. Distúrbios do sono e a odontologia. São Paulo: Santos; 2010.

17. MARCUS CL. Sleep-disordered breathing in children. American Journal of Respiratory and Critical Care Medicine, 2000; 164: 16-30.

18. MARSON A, TESSITORE A, SAKANO E, et al. Efetividade da fonoterapia e proposta de intervenção breve em respiradores orais. Revista CEFAC, 2012; 14(6): 1153-1166.

19. MARTINS AB, TUFIK S, MOURA SMGPT. Síndrome da apneia e hipopneia obstrutiva do sono. Fisiopatologia. Jornal Brasileiro de Pneumologia, 2007; 33(1): 93-100.

20. MENEZES VA, LEAL RB, PESSOA RS, et al. Prevalência e fatores associados à respiração oral em escolares participantes do projeto Santo Amaro-Recife, 2005. Revista Brasileira de Otorrinolaringologia, 2006; 72(3): 394399.

21. NISHIMURA CM, GIMENEZ SRML. Perfil da fala do respirador oral. Revista CEFAC, 2010; 12(3): 505-208.

22. PIGNATARI SSN, ANSELMO-LIMA WT. Tratado de otorrinolaringologia. 3rd ed. Rio de Janeiro: Elsevier, 2018; $991 \mathrm{p}$.

23. POPOASKI C, MARCELINO TF, SAKAE TM, et al. Avaliação da qualidade de vida em pacientes respiradores orais. Arquivos Internacionais de Otorrinolaringologia, 2012; 16(1): 74-81.

24. RAMOS RTT, DALTRO CHC, GREGÓRIO PB, et al. SAHOS em crianças: perfil clínico e respiratório polissonográfico. Revista Brasileira de Otorrinolaringologia, 2006; 72(3): 355-361.

25. RIBEIRO GCA, SANTOS ID, SANTOS ACN, et al. Influence of the breathing pattern on the learning process: a systematic review of literature. Brazilian Journal of Otorhinolaryngology, 2016; 82(4): 466-478.

26. RIBEIRO ML, PINTO JA. Qualidade de vida no respirador oral: avaliação sistemática em crianças de 6 a 12 anos, atendidas em centro de referência da UFMG. Minas Gerais. Dissertação - Universidade Federal de Minas Gerais, 2006.

27. RUEHLAND WR, ROCHFORD PD, O'DONOGHUE FJ, et al. The new AASM criteria for scoring hypopneas: Impact on the apnea hypopnea index. SLEEP, 2009; 32(2):150-157.

28. TERSE-RAMOS R. Síndrome da Apneia Obstrutiva no Sono na Infância. Pulmão RJ, 2013; 22(3): 26-30.

29. TESSITORE A. Alterações oromiofuncionais em respiradores orais. In: FERREIRA LP, BEFI-LOPES DM, LIMONGI SCO, et al. Tratado de Fonoaudiologia. São Paulo: Roca, 2004; 1076p.

30. VIANNA CIO, SUZUKI HS. Paralisia cerebral: análise dos padrões da deglutição antes e após intervenção fonoaudiológica. Revista CEFAC, 2011; 13(5): 790-800.

31. ZANCANELLA E, HADDAD FM, OLIVEIRA LAMP, et al. Obstructive sleep apnea and primary snoring: diagnosis. Brazilian Journal of Otorhinolaryngology, 2014; 80(1 Suppl I): 1-16. 